\title{
Por una mejora de la usabilidad y utilidad en las herramientas de postedición
}

\author{
Upgrading Usability and Utility Features for Shaping \\ Post-Edition Tools
}

\section{Coral Diez CARBAJO}

Universidad de Salamanca

coral.diez@usal.es

Recibido: julio 2018. Revisado: septiembre 2018. Aceptado: octubre 2018.

Resumen: El desarrollo de las tecnologías ha posibilitado la creación de herramientas de apoyo a la postedición cada vez más completas a nivel técnico, centradas en facilitar la tarea al humano y mejorar la experiencia de los usuarios. Con el fin de definir las funcionalidades necesarias en una herramienta de postedición asistida, se han seleccionado ciertas características funcionales ya existentes en herramientas de traducción asistida por ordenador y motores de traducción automática que los expertos consideran útiles para poseditar. Asimismo, se analizan los resultados de interacción persona-ordenador y de diseño de interfaces orientadas a mejorar la experiencia del usuario para aplicarlos al desarrollo de herramientas de postedición usables. Con dicho análisis, se pretende ganar calidad y rapidez en el proceso de postedición y enriquecer las condiciones de trabajo de este perfil profesional emergente.

Palabras clave: postedición; herramienta de postedición; usabilidad; utilidad; desarrollo de herramientas; diseño de interfaces. 


\begin{abstract}
Technological advances have led to the development of ever more comprehensive translation and post-edition support tools focusing on facilitating the human's task and improving the users' experience when interacting with machines. This article seeks to specify the most needed functionalities to create a comprehensive assisted post-edition tool by selecting some of the existing functions highlighted as useful by experts working with computer assisted and machine translation tools. Furthermore, an analysis of the most relevant aspects of human-computer interaction and interface design will follow, focused on enhancing user experience and improving post-edition tools usability. The aim of this study is to prioritize the quality and speed of the post-edition process by improving humans' working conditions of this emerging professional profile.
\end{abstract}

Key words: post-edition; post-edition tool; usability; utility; tool development; interface design.

\title{
1. INTRODUCCIÓN
}

En el mundo globalizado e interconectado en el que vivimos necesitamos recurrir a los avances tecnológicos para aumentar la productividad y lidiar con la gran cantidad de documentos que precisan traducción, ya que nos permiten ganar rapidez sin dejar de lado la calidad que cualquier texto requiere para cumplir con su función (Allen 2001; Guerberof Arenas 2008; O'Brien 2012; Dillinger 2014). Como todo apunta a que esta estrecha colaboración del humano con la máquina será el futuro de nuestra profesión (Robert 2010), se hace evidente la necesidad de desarrollar y diseñar herramientas informáticas que favorezcan nuestra participación crítica en el proceso de traducción con tecnologías.

Hasta hace unos años, no existían herramientas propiamente desarrolladas para poseditar contenido generado por motores de traducción automática; el trabajo se realizaba desde interfaces de traducción asistida por ordenador (TAO). Estas herramientas ayudan al humano proponiendo resultados de traducción basados en memorias de traducción y bases de datos terminológicas -ambas creadas a partir del trabajo previamente realizado por personas- que permiten a los traductores reciclar el contenido repetido en diferentes documentos (Torres del Rey y Morado Vázquez 2015). Más tarde, comenzaron a desarrollarse interfaces de edición de traducciones procedentes no solo de herramientas de traducción asistida, sino también de motores de traducción automática (TA) en los que la traducción se realiza directamente por una herramienta de software específicamente desarrollada para ello y que parte de glosarios y reglas («traducción automática basada en reglas»), grandes corpus y fórmulas estadísticas ("traducción automática estadística») y/o técnicas propias de la inteligencia artificial y del procesamiento del lenguaje natural («traducción automática neuronal») para encontrar el equivalente más frecuente del texto fuente en la lengua meta.

Coral DIEZ CARBAJO Por una mejora de la usabilidad y utilidad en las herramientas de postedición
CLINA

vol. 4-2, December 2018, 123-140

elSSN: 2444-1961

Ediciones Universidad de Salamanca - CC BY-NC-ND 
Simplificando, la principal diferencia entre las herramientas de traducción asistida por ordenador y de traducción automática radica en quién "compone» el texto que devuelve la herramienta. En el primer caso, los segmentos se componen de acuerdo con el porcentaje de similitud que comparten con aquellos que ya se encuentren en las memorias de traducción, que recogen traducciones generadas por personas, por lo que los resultados se basan en el trabajo realizado por el humano. Por otra parte, en el caso de la traducción automática, es el propio motor el que compone las traducciones reorganizando, juntando y mezclando secciones de textos traducidos por humanos y utilizando reglas, algoritmos o cómputos matemáticos. No obstante, en la actualidad, los límites entre ambas herramientas no están tan definidos, ya que las tecnologías más novedosas aúnan ambas técnicas: por lo general, en un primer momento, se parte del contenido existente en las memorias para devolver resultados de traducción previamente traducidos por humanos y, a continuación, se completan los segmentos sin resultados en las memorias con traducciones automáticas realizadas por motores (Reinke 2013; O'Brien y Moorkens 2014).

En la última década se han creado herramientas de traducción automática de código abierto y programas que gozan de precios menos prohibitivos (o que incluso ofrecen algunos servicios de manera gratuita), popularizando su uso, antes restringido a las grandes empresas o corporaciones (Flórez 2010). Asimismo, factores como los avances en las investigaciones teóricas, los progresos en ciencias computacionales, los nuevos conocimientos sobre programación, los estudios sobre el procesamiento del lenguaje natural, las mejoras en la capacidad de almacenamiento de datos o la creación de nuevos algoritmos, entre otros (Kay 1997) han influido de manera positiva en la creación de herramientas de traducción automática más avanzadas, que utilizan redes neuronales y técnicas propias de la inteligencia artificial para producir resultados de traducción en bruto más precisos.

Hasta hace unos años, los traductores poseditaban desde estas plataformas híbridas de traducción asistida y automática. Sin embargo, a medida que la traducción asistida y automática con postedición humana creció en popularidad tanto en el mundo académico como en el empresarial, se mostró imprescindible el desarrollo de una herramienta especialmente diseñada para asistir el trabajo del poseditor. Esto es debido a que traducir y poseditar son acciones que requieren capacidades parcialmente diferentes. El poseditor parte de dos textos originales: el texto origen y el texto «en bruto" pretraducido por herramientas de traducción asistida y automática, para revisarlos de acuerdo con unas normas específicas a fin de atenerse a unos estándares de calidad -generalmente, se distingue entre full post-editing ("postedición completa») y light post-editing («postedición rápida») (TAUS 2010).

Por otra parte, recientemente se ha producido un giro en la manera de enfocar el desarrollo de las herramientas de traducción automática desde las que se puede poseditar. Mientras que, antes, la mejora de las herramientas se centraba en ciertas

Coral DIEZ CARBAJO Por una mejora de la usabilidad y utilidad en las herramientas de postedición 
especificaciones técnicas para modernizar el funcionamiento de la máquina, ahora, se está empezando a tener en cuenta la interacción del humano con las interfaces para facilitar el trabajo a los profesionales que poseditan con ellas (Moorkens y O'Brien 2017). Este cambio de enfoque no es exclusivo de las herramientas de postedición, sino que también puede observarse en el desarrollo de herramientas de traducción asistida y automática en los últimos años. Dicho cambio de paradigma en la creación de herramientas viene motivado por la insatisfacción que los usuarios sienten al poseditar desde las plataformas que no se han creado específicamente para ello (Moorkens, O’Brien y Vreeke 2016), por lo que de las lecturas realizadas se concluye que todavía queda mucho por mejorar en el desarrollo de herramientas de postedición «útiles» y «usables» (términos explicados en detalle en los apartados 2.1 y 2.2 de este artículo) que favorezcan la experiencia de los usuarios.

Para conseguir una herramienta práctica y eficaz se determinan, en primer lugar, las características funcionales que aportan «utilidad» a las herramientas de postedición asistida y así asegurar que el poseditor puede completar todo el proceso de trabajo desde la misma herramienta, lo que maximiza el rendimiento del humano. Asimismo, se concretan los aspectos que benefician la «usabilidad» de las mismas, al facilitar su utilización, mejorar la experiencia del usuario y favorecer un aprendizaje más intuitivo. Muchas de estas nuevas herramientas que persiguen facilitar la tarea de postedición al humano, todavía en desarrollo, se están creando tras realizar estudios de usabilidad con usuarios para conocer sus experiencias y analizar las dificultades que encuentran al usar estas máquinas. Al tener en cuenta tanto la utilidad como la usabilidad se crean herramientas prácticas que mejoren las condiciones humanas, en lugar de focalizar los esfuerzos exclusivamente en el desarrollo técnico (Winograd 1997: 155).

Con el fin de mitigar los puntos débiles que los poseditores encuentran al interactuar con las herramientas y conseguir que aumente la satisfacción de los humanos, las ciencias computacionales están uniendo sus fuerzas con la psicología, la lingüística o el diseño gráfico en la búsqueda de herramientas que cubran las expectativas de los usuarios, aumenten su productividad y sean fáciles de usar. A pesar de que no resulta fácil estudiar, medir y controlar las necesidades cognitivas, técnicas y visuales de los usuarios, es indispensable investigar en diseño de interacción, utilidad y usabilidad para diseñar herramientas más atractivas y manejables. La transversalidad que están alcanzando estos estudios se justifica por las consecuencias directas encontradas en el aumento del rendimiento, eficacia y productividad de los poseditores cuando las experiencias de los usuarios son positivas (Sukholeyster 2014). De este modo, los poseditores contarían con una interfaz agradable, adecuada a sus necesidades, que les ayude a entender las relaciones de operatividad persona-ordenador y que les ofrezca características que potencien sus capacidades y reduzcan sus limitaciones, por lo que estarían más estimulados y se sentirían más cómodos en su lugar de trabajo, lo que, a su vez, aumentaría la productividad y recompensaría su creatividad, haciendo de la postedición una tarea más grata y sencilla.

Coral DIEZ CARBAJO Por una mejora de la usabilidad y utilidad en las herramientas de postedición
CLINA

vol. 4-2, December 2018, 123-140

elSSN: 2444-1961

Ediciones Universidad de Salamanca - CC BY-NC-ND 
Por ello, con el fin de facilitar el desarrollo de una herramienta de asistencia a la postedición que ayude al humano a realizar su tarea, en el apartado 2 de este artículo se analizan, en primer lugar, las características funcionales que han demostrado ser de utilidad a la hora de poseditar (tanto previas como durante y después del proceso de postedición) y, en segundo lugar, los aspectos de interacción que aportan usabilidad para poseditar. Finalmente, a modo recapitulativo, se explica la relación existente entre las características funcionales y los aspectos de interacción.

\section{ANÁLISIS}

Todavía se puede mejorar mucho la utilidad y la usabilidad de una herramienta de postedición cuando se entienden dichas herramientas como instrumentos que fomentan la tarea del humano (Nunes Vieira y Specia 2011; Moorkens, O'Brien y Vreeke 2016). Hasta hace unos años, lo más común era poseditar directamente en herramientas de traducción asistida, trabajando con los segmentos generados a partir de memorias de traducción o de manera automática. No obstante, tras haberse probado la insatisfacción que experimentaban los traductores al poseditar desde estas plataformas (Flórez 2012; Guerberof, Depraetere y O'Brien 2012; O’Brien y Moorkens 2014) y debido a que un poseditor no se enfrenta simplemente a la revisión de un texto traducido automáticamente, sino que lidia con una gran cantidad de diversas tareas (como se explica en el apartado 2.1), se comenzaron a crear herramientas interactivas de gestión lingüística para la traducción automática y la postedición que asisten al humano en la realización de sus labores durante todo el proceso lingüístico: gestión del proyecto, entrenamiento de los motores, limpieza de memorias, postedición, revisión de los estándares de calidad y retroalimentación de la herramienta, entre otros.

La creación de una herramienta de postedición asistida interactiva tan completa y compleja requiere una gran inversión en investigación previa a su desarrollo. Este artículo recoge ciertas características funcionales que profesionales e investigadores que han trabajado con herramientas de traducción consideran de utilidad para el humano -ya sea gracias a su propia práctica o a través de la realización de encuestas- (Melby 1997; Flórez 2012; Sánchez Martínez 2012; Guerberof, Depraetere y O’Brien 2012; Reinke 2013; Silva 2014; O'Brien y Moorkens 2014; de Jesus Martins y de Medeiros Caseli 2015; Moorkens, O’Brien y Vreeke 2016). De este modo, esas características funcionales se pueden incluir en la construcción de una herramienta de postedición asistida y así asegurar que dicha herramienta es útil para el poseditor. Por otro lado, para determinar ciertos aspectos de interacción que favorecen la usabilidad de dichas herramientas, se han aplicado al desarrollo concreto de estas herramientas de postedición asistida las conclusiones de estudios genéricos sobre usabilidad, diseño de interfaces, experiencia de usuario, accesibilidad o interacción persona-ordenador

Coral DIEZ CARBAJO Por una mejora de la usabilidad y utilidad en las herramientas de postedición 
(Nielsen 1995; Morville 2004; Gutiérrez y Restrepo y Martínez Normand 2010; Garrett 2011; Nielsen 2012; Kuniavsky 2012; Rodríguez Vázquez y Torres del Rey 2014; Meyer 2017).

\subsection{Características funcionales de utilidad para posteditar}

\subsubsection{Características funcionales previas al proceso de postedición}

Existen numerosas características funcionales que pueden desarrollarse para crear una herramienta que asista al usuario-poseditor durante la realización de todas las tareas de su trabajo. Comenzando por la etapa previa a la propia postedición, una herramienta de postedición asistida debe permitir que el poseditor entrene los motores de traducción automática cada vez que haya un proyecto nuevo, partiendo de archivos que compartan la temática del texto que se necesita traducir. En la actualidad existen numerosas herramientas que integran traducción automática. Sin embargo, los motores de dichas herramientas suelen ser generales, su entrenamiento no parte de un corpus específico con información propia del cliente. Al alimentar una herramienta de traducción automática con la tipología de textos que va a tener que traducir, se puede influir en la obtención de resultados para que sean más acertados, sigan las características formales del encargo y se adapten a la terminología propia del cliente, por lo que el poseditor tendrá que realizar menos cambios. Asimismo, al personalizar la herramienta de postedición asistida en cada proyecto, se pueden incluir instrucciones relativas al grado de postedición (postedición rápida -sin errores, pero generalmente superficial- o postedición completa -en la que se postedita con más profundidad para conseguir una calidad humana) con el que poseditar cada encargo debido a la multitud de variantes que pueden influir: tiempo, presupuesto o requisitos especificados por el propio cliente.

Igualmente, al crear nuevas herramientas de postedición asistida útiles, es fundamental que sean capaces de leer, importar y exportar al menos la misma cantidad de formatos de archivos diferentes que las herramientas de traducción asistida y automática ya existentes en el mercado ya que, el simple hecho de ser capaces de introducir los documentos en la herramienta es el primer paso para poder trabajar con ella. En los últimos años, los desarrolladores de herramientas de traducción asistida y automática han centrado gran parte de sus esfuerzos en compatibilizar una amplia gama de formatos de archivos con el trabajo con herramientas de traducción. Gracias a eso, a pesar de que todavía seguimos encontrando dificultades al trabajar con documentos en .pdf, podemos beneficiarnos de estas herramientas no solo para traducir o poseditar los diferentes documentos con la ayuda de estas tecnologías, sino también para mantener el formato y la maquetación en multitud de formatos.

Coral DIEZ CARBAJO Por una mejora de la usabilidad y utilidad en las herramientas de postedición 
En consonancia con el aprovechamiento de todos los avances tecnológicos desarrollados hasta la fecha, una plataforma de postedición útil debe integrar un sistema híbrido de generación de pretraducciones que, como ocurre generalmente, utilice primero los resultados provenientes del contenido existente en las memorias -a partir de la comparación por distancia de edición- y después, traduzca con motores de traducción automática basada en reglas, estadística o neuronal los segmentos con un nivel de coincidencia insatisfactorio. En este caso, para facilitar el trabajo al poseditor, la literatura (véanse, por ejemplo, O'Brien y Moorkens 2014 o Reinke 2013) sugiere la necesidad de que se indique la procedencia del segmento -memorias o generación automática- ya que la tipología de errores que comete un humano (información de las memorias) es diferente al tipo de fallos que encontramos en los segmentos producidos de manera automática (si bien es cierto que, en el caso de la traducción automática neuronal, los errores son cada vez más similares a los cometidos por los humanos). Finalmente, sería de utilidad la visualización del porcentaje de fiabilidad y coincidencia con la información de las memorias para guiar al humano en la toma de decisiones. Esta característica funcional, a pesar de estar completamente implantada en las herramientas de traducción asistida, todavía se encuentra en desarrollo en las herramientas de traducción automática -y, más concretamente, en los motores de traducción automática neuronal.

Una última característica funcional que proponemos antes del proceso de postedición sería la posibilidad de "preeditar» desde la propia plataforma, adaptando los textos fuentes, antes de traducirlos de manera automática, para dotarlos de claridad y sencillez de redacción, precisión y coherencia terminológica, eliminar las ambigüedades e inexactitudes en el texto original, y corregir los errores del texto origen (Rico s.f.). Al preeditar, se pretende eliminar los obstáculos que la máquina se pueda encontrar y que puedan causar dificultades de "comprensión» a la herramienta para así mejorar la calidad de las pretraducciones devueltas. De este modo, se crean textos con lenguaje controlado para máquinas que elimina las ambigüedades inherentes al lenguaje humano (O’Brien 2006: 2).

Es útil preeditar un texto cuando la pretraducción del texto preeditado es más fácil de poseditar que la postedición del texto en bruto traducido automáticamente sin preedición, por lo que el humano reducirá el esfuerzo y el tiempo empleados en completar el encargo. Para comprobar si merece la pena preeditar un texto, se puede calcular su grado de "traducibilidad automática» (Ramírez Polo 2012: 199) aportando valores negativos a los aspectos que presentan obstáculos al motor de traducción automática como faltas de ortografía, voz pasiva, expresiones idiomáticas, estructuras ambiguas, frases de mucha longitud, elipsis, metáforas o dependencia entre frases (Berth y Gdaniec 2001). Por cada obstáculo detectado, el texto es menos traducible automáticamente y, en los casos en los que el valor del texto esté por debajo de un mínimo preestablecido, se estima necesario preeditar para eliminar esas dificultades y facilitar

Coral DIEZ CARBAJO Por una mejora de la usabilidad y utilidad en las herramientas de postedición 
la postedición. Por ello, de las lecturas realizadas se desprende que una herramienta de postedición asistida completa debería mostrar información acerca de la traducibilidad automática del texto, en la ventana desde la que se pueda preeditar, para que el poseditor decida si le interesa preeditar el texto antes de traducirlo automáticamente.

\subsubsection{Características funcionales durante el proceso de postedición}

Una vez se vaya a poseditar es imprescindible que en una herramienta de postedición, las pantallas de edición permitan ver el texto fuente y el meta de manera simultánea y cuenten con características funcionales que nos ayuden a seguir interactivamente ambos textos. El ejemplo más común es la desaparición automática de los segmentos que ya se han completado. De este modo, al confirmar que la postedición de un segmento se ha terminado, la interfaz dejaría de mostrar ese segmento para enseñar el siguiente con el que debemos trabajar, de modo que el poseditor no perdería tiempo avanzando el texto. Esta característica puede entenderse como una ventaja o como un impedimento, según las necesidades del poseditor o las características del encargo; por ello, como se indica en el apartado 2.2, es importante que el humano pueda tomar decisiones acerca de la customización de la herramienta. En este sentido, existen otras características funcionales de interacción avanzadas ya desarrolladas en las herramientas de postedición asistida más novedosas, como el emparejamiento de palabras origen con su equivalente en destino por medio de subrayados o colores. Esta característica funcional permite al poseditor seguir los segmentos en ambas lenguas sin perderse.

Además de las características funcionales ya existentes anteriormente señaladas, expertos como O'Brien y Moorkens 2014 o Meyer 2017 estiman necesario el desarrollo de otras, como la posibilidad de que sea el propio poseditor el que cree y añada atajos de teclado avanzados para cambiar la puntuación, las mayúsculas y minúsculas de las palabras, su género o su número, o deshacer las sugerencias propuestas de manera automática, así como permitir el arrastre de palabras o etiquetas dentro del texto meta. Como cada humano puede tener preferencias diferentes y cada herramienta puede estar desarrollada de manera que sus errores más recurrentes sean distintos (siglas, nombres propios, concordancia de género o número, posicionamiento sustantivo-adjetivo, entre otros), un poseditor puede necesitar atajos de teclado diversos dependiendo de las instrucciones de cada proyecto y de sus gustos y necesidades personales. Al incluir esta característica funcional, las herramientas serían más útiles al hacerse más accesibles y permitir la realización de las tareas más recurrentes no solo con el ratón, sino también con el teclado.

Para que las herramientas de postedición asistida de nueva creación sean más interactivas es indispensable desarrollar características funcionales que favorezcan el

Coral DIEZ CARBAJO Por una mejora de la usabilidad y utilidad en las herramientas de postedición
CLINA

vol. 4-2, December 2018, 123-140

elSSN: 2444-1961

Ediciones Universidad de Salamanca - CC BY-NC-ND 
autoaprendizaje de la máquina. En este sentido, destaca la importancia de las sugerencias de escritura predictiva para autocompletar segmentos con la información previamente corregida por el humano. De esta forma, como ya ocurre en algunas herramientas TAO, el usuario iría recibiendo sugerencias actualizadas y en tiempo real de las combinaciones de palabras con una mayor probabilidad de ser las siguientes que el humano va a tener que teclear, para que el poseditor solo tenga que seleccionar las sugerencias correctas, lo que le ahorraría el tiempo de teclear el resto del segmento. Esta característica funcional se perfeccionaría si la herramienta fuera capaz de aprender y reentrenarse, extrayendo términos y alimentándose con la alineación de los nuevos segmentos ya confirmados. Así, se autopropagarían los cambios realizados por el humano mientras se trabaja con la herramienta, garantizando que las sugerencias propuestas se adapten a las correcciones que el humano va realizando conforme trabaja desde ella. El desarrollo de las funciones de autoaprendizaje de las herramientas de postedición resulta de utilidad no solo a la hora de aportar sugerencias de autocompletado adaptadas a las preferencias del poseditor, sino también para evitar que el humano corrija el mismo error varias veces en el mismo proyecto, eliminando la sensación de pesadez y monotonía que tradicionalmente se asocia a la postedición de errores repetitivos y recurrentes.

Por otra parte, se necesita el desarrollo de una función de ayuda dentro de la pantalla de edición para recuperar las pautas y las normas del encargo, y que el poseditor pueda asegurarse de que se cumplen los requisitos del proyecto y de que se siguen la guía de estilo y la de postedición impuestas por el cliente para cada encargo. Asimismo, dentro esta misma pantalla, resulta de utilidad la posibilidad de realizar búsquedas avanzadas de terminología o segmentos para determinar cómo se ha procedido en situaciones similares y así asegurar la coherencia interna del proyecto, como ya ocurre en las herramientas de TAO. Esta función de gestión y reconocimiento terminológico puede complementarse con el despliegue de listas con alternativas de traducción para que el poseditor pueda seleccionar su opción favorita. De esta forma, también se reforzarían las ideas de la necesidad de implantar atajos de teclado avanzados para desplegar estas opciones y seleccionar la correcta con rapidez y de la mejora de la accesibilidad, ya que se posibilitaría el acceso a esta información terminológica de manera más cómoda.

\subsubsection{Características funcionales después del proceso de postedición}

Finalmente, una vez terminado el encargo de postedición, sería oportuno que se pudieran recopilar datos acerca del proceso de trabajo, como el tiempo y el esfuerzo empleado por el humano para poseditar. De este modo, se podrían estimar el tiempo y esfuerzo que ese mismo poseditor empleará en proyectos futuros, lo que ayudará al

Coral DIEZ CARBAJO Por una mejora de la usabilidad y utilidad en las herramientas de postedición 
humano a planificar su flujo de trabajo y a emitir facturas conforme a la labor realizada. Al mismo tiempo, contar con este tipo de información nos ayudaría a asegurarnos de que los recursos empleados en poseditar son inferiores a los que se utilizarían en traducir ese mismo documento, arrojando un poco de luz sobre el esfuerzo y el tiempo real que un poseditor necesita para conseguir la calidad deseada.

\subsection{Aspectos de interacción que aportan usabilidad para posteditar}

Recordemos que, para que una herramienta de postedición asistida sea práctica, además de ser «útil» y permitir que el humano realice las tareas que necesita para completar su trabajo, tiene que ser «usable», ya que de nada sirve que una herramienta sea completa con respecto a las características funcionales que ofrece, si esta no se puede usar por ser demasiado complicada, confusa en su uso, incómoda o poco intuitiva. Por ello, el diseño de una herramienta usable debe ser familiar y fácil de entender para asegurar que la curva de aprendizaje del usuario sea lo más corta e «indolora» posible (Morville 2004).

A principios del siglo XXI se empezaron a diseñar herramientas pensando en la experiencia de los usuarios y el tipo de interacción que el humano iba a tener con la máquina. Para que los desarrolladores comprendieran las necesidades de los usuarios se evaluaron los aspectos de interacción que aportaban usabilidad a las herramientas. Uno de los métodos de evaluación de la usabilidad que se comenzaron a utilizar fue la observación del comportamiento de los usuarios mientras interactuaban con las máquinas y la realización de encuestas y entrevistas en las que se pedía a los usuarios que relataran su experiencia. Partiendo de esas respuestas, los desarrolladores mejoraban la herramienta para que le resultara más intuitiva al usuario y trataban de eliminar los obstáculos con los que se había encontrado. Este proceso se repetiría hasta conseguir una herramienta que los humanos pudieran utilizar con facilidad, lo que incrementaría el rendimiento y la satisfacción del usuario, y reduciría costes y tiempo de postedición y de aprendizaje del manejo de la herramienta.

Con respecto a esto, Morville (2004) señala que, en las últimas décadas, las empresas han comenzado a valorar la experiencia de los usuarios en el diseño de herramientas informáticas. Sin embargo, como en ocasiones es difícil prestar atención a los diferentes factores que mejoran la experiencia de usuario, Morville definió las seis facetas que influyen en el diseño de herramientas con el fin de ayudar a las empresas a encontrar un equilibrio entre todas ellas y, a su vez, favorecer la experiencia de los usuarios meta de la plataforma o herramienta, ya que no todos los productos o empresas ponderarán sus prioridades de igual manera: algunos preferirán hacer que su producto sea más deseable, otros que sea más creíble o accesible, por ejemplo. Siguiendo las indicaciones de Morville (2004), una herramienta debe ser (1) «usable» 
(usable): el producto debe ser sencillo de usar, resultarle familiar al usuario y se debe aprender su funcionamiento con facilidad; (2) «útil» (usefu): el producto debe servir para cumplir con el propósito con el que se creó1; (3) «deseable» (desirable): la herramienta debe cumplir con las características visuales que hagan que sea lo suficientemente atractiva y fácil de entender como para que el usuario quiera trabajar con ella; (4) «accesible» (accessible): el servicio debe estar diseñado de tal forma que incluso los usuarios con algún tipo de discapacidad puedan utilizarlo; (5) «manejable» (findability): el usuario debe poder encontrar la información que necesita y entender cómo navegar por la estructura del producto; y (6) "creíble» (credible): el usuario debe poder confiar en la información que esa plataforma le presenta.

A pesar de que la mayoría de los estudios realizados al respecto tratan la usabilidad de las herramientas informáticas en general, los resultados de dichos estudios se pueden extrapolar al caso del desarrollo de herramientas de postedición fáciles de usar. En primer lugar, para que una herramienta de postedición asistida pueda usarse debe ser «accesible»: el poseditor debería poder elegir cómo interactuar con la herramienta. En este sentido, a lo largo de este artículo se han ido indicando ejemplos, como la inclusión de atajos de teclado para realizar las acciones más recurrentes en cada etapa del proceso de postedición, como pueden ser cambiar la concordancia del género o número o desplegar listas de sugerencias de traducción o terminología en lugar de recurrir al ratón. Otros ejemplos de diferentes modos de accesibilidad a la hora de interactuar con una herramienta de postedición serían la posibilidad de arrastrar términos o conjuntos de palabras seleccionándolas con una pantalla táctil o de contar con la lectura en voz alta del texto fuente y la pretraducción automática.

Además, es fundamental que una herramienta de postedición asistida sea lo suficientemente «intuitiva» (o manejable) para que un usuario pueda aprender a utilizarla con rapidez, entender y recordar su estructura, y utilizarla a nivel avanzado con facilidad. El poseditor debería intuir qué acción puede realizar en cada una de las pantallas y etapas del proceso (creación del proyecto, preedición, postedición, gestión terminológica, exportación del texto poseditado, análisis de los datos una vez completado el encargo, etc.) y cómo moverse dentro de la interfaz y encontrar las características funcionales que necesite en cada momento para realizar su trabajo (personalizar los motores de traducción automática, encontrar las instrucciones de postedición del proyecto concreto, generación de vistas previas del documento final, propuestas de sugerencias para completar segmentos, despliegue de listas con opciones de traducción alternativas...).

Asimismo, una herramienta de postedición práctica debe «anticiparse a los problemas» (Nielsen 1995) con los que el usuario se pueda encontrar para prever la

1. Esta característica se relaciona con la propuesta original de Nielsen (1995) y Nielsen (2012), seguida en este trabajo y que diferencia entre «utilidad» y «usabilidad».

Coral DIEZ CARBAJO Por una mejora de la usabilidad y utilidad en las herramientas de postedición 
información que el humano puede necesitar y evitar que cometa un error. Por ello, en una herramienta de postedición asistida es fundamental nombrar los botones de manera relevante y de acuerdo con los estándares (como: «inicio", "abrir archivo", «actualizar memorias» o «nuevo proyecto».) para, a su vez, facilitar la adecuación de su utilización a los usuarios que estén familiarizados con trabajar desde herramientas de traducción asistida o automática. También evita que surjan problemas el añadir cuadros aclarativos que se desplieguen al pasar el ratón por encima de los propios botones ya que así, el usuario puede conocer las repercusiones que tiene seleccionarlo y considerar, con más información, si necesita que se desencadene esa acción o no. De todos modos, incluso cuando se expliquen las consecuencias de activar una tarea, sería imprescindible que se permitiera la opción de hacer y deshacer cualquier acción y se mostrara un cuadro de confirmación antes de ejecutar acciones que no se puedan deshacer. Finalmente, una vez se haya cometido el fallo, una herramienta diseñada con el fin de facilitar el trabajo al poseditor debe ofrecer cuadros de errores con soluciones útiles y claras, que le ayuden a solventar el problema que ha causado.

Por otra parte, relacionado con la accesibilidad, es necesario que el poseditor pueda personalizar la herramienta según sus gustos, sus preferencias o sus capacidades físicas. Esta "customización» es fundamental en todos los niveles del proceso de trabajo. El poseditor debería ser capaz de elegir y seleccionar qué características funcionales le resultan de utilidad y cuáles le obstaculizan su trabajo (despliegue de grandes listas de sugerencias de traducciones alternativas, visualización de más o menos contexto del segmento con el que se está trabajando, entre otros). También, debería poder configurar la visualización de la información de la pantalla -permitiéndole que oculte, muestre o modifique la posición de las ventanas, cambie la configuración de colores o el tamaño de letra, entre otros- para visualizar toda la información que el poseditor necesita en cada momento y no obligarle a depender de su memoria a corto o largo plazo.

Por último, no hay que minusvalorar la «planificación de la estructura» de la herramienta para diseñarla de manera coherente y cerciorarse de que se respetan los estándares, se dispone la información siguiendo un orden lógico y se utiliza un lenguaje sencillo e intuitivo que permita a cualquier usuario interactuar con ella (Sukholeyster 2014). Por ello, en el caso de una herramienta de postedición, es evidente la necesidad de colocar las diferentes pestañas (creación del proyecto, entrenamiento de motores, traducción automática, preedición, postedición, exportación del texto poseditado, análisis de los datos una vez completado el encargo, entre otras) siguiendo el orden temporal del proceso completo del que se tiene que encargar el poseditor, a pesar de que el humano pueda decidir saltarse alguno de los pasos en ciertos encargos. Así mismo, al desarrollarse la herramienta, el humano debe poder acceder a cada característica funcional en el momento en el que la vaya a necesitar: acceder a la información relativa a la traducibilidad automática desde la pestaña de preedición, tener acceso a la gestión terminológica desde la pestaña de postedición, etc.

Coral DIEZ CARBAJO

Por una mejora de la usabilidad y utilidad en las herramientas de postedición
CLINA

vol. 4-2, December 2018, 123-140

elSSN: 2444-1961

Ediciones Universidad de Salamanca - CC BY-NC-ND 
En esta línea, es esencial prestar atención a aspectos del «diseño gráfico»-como usar diferentes colores, texturas, fuentes o tamaños de letras- que mejoren la estética de la interfaz, haciendo que sea más agradable trabajar con ella y más fácil encontrar la información que necesite el poseditor al incrementar la legibilidad, focalizar la atención de los usuarios en los puntos más importantes o marcar las relaciones jerárquicas de los componentes de la herramienta. Por ejemplo, en una herramienta de postedición asistida se podrían oscurecer las características funcionales que el poseditor no pueda utilizar en esa etapa del proyecto; resaltarse los términos que estén incluidos en la base terminológica o presentar en colores diferentes los segmentos provenientes de las memorias de traducción y los generados con motores de traducción automática.

\subsection{Relación entre las características funcionales y los aspectos de interacción}

La "oficina» del poseditor es la información que recoge a través de la pantalla del ordenador, por lo que es fundamental que el usuario se sienta cómodo utilizando la interfaz con la que trabaja. Como se muestra en el siguiente cuadro recapitulativo (Tabla 1), la implantación de las características funcionales que se han ido mencionando en este artículo repercute en la manera en la que el humano puede interactuar con la herramienta de postedición al beneficiar alguna de las facetas mencionadas por Morville (2004).

\begin{tabular}{|l|c|}
\hline Característica funcional & Aspecto de interacción \\
\hline $\begin{array}{l}\text { Entrenamiento de los motores de traducción automática en } \\
\text { cada encargo }\end{array}$ & Accesible (customización) \\
\hline Inclusión de instrucciones propias de cada proyecto & Accesible (customización) \\
\hline $\begin{array}{l}\text { Importación y exportación de una amplia variedad de } \\
\text { formatos de archivos }\end{array}$ & Accesible \\
\hline $\begin{array}{l}\text { Integración de un sistema híbrido de generación de } \\
\text { pretraducciones }\end{array}$ & Útil \\
\hline $\begin{array}{l}\text { Información de la procedencia de los segmentos } \\
\text { pretraducidos }\end{array}$ & Creíble \\
\hline $\begin{array}{l}\text { Visualización del porcentaje de fiabilidad y coincidencia de } \\
\text { la pretraducción }\end{array}$ & Creíble \\
\hline Incorporación de una pantalla de preedición & Útil \\
\hline Cálculo de la traducibilidad automática & Útil \\
\hline
\end{tabular}

Coral DIEZ CARBAJO Por una mejora de la usabilidad y utilidad en las herramientas de postedición 


\begin{tabular}{|c|c|}
\hline Característica funcional & Aspecto de interacción \\
\hline Visualización simultánea de texto fuente y meta & Usable \\
\hline $\begin{array}{l}\text { Desaparición automática de los segmentos ya } \\
\text { completados }\end{array}$ & $\begin{array}{l}\text { Útil y Accesible } \\
\text { (customización) }\end{array}$ \\
\hline Emparejamiento interactivo de palabras origen y destino & Accesible (customización) \\
\hline Creación de atajos de teclado avanzados & Accesible (customización) \\
\hline Arrastre de términos desde una pantalla táctil & Accesible (customización) \\
\hline Lectura en voz alta del texto & Accesible (customización) \\
\hline Autoaprendizaje de la herramienta & Deseabilidad \\
\hline Sugerencias de escritura predictiva & $\begin{array}{l}\text { Deseabilidad y Accesible } \\
\text { (customización) }\end{array}$ \\
\hline Función de ayuda & Manejable \\
\hline Búsquedas terminológicas avanzadas & $\begin{array}{l}\text { Útil y Accesible } \\
\text { (customización) }\end{array}$ \\
\hline Despliegue de listas con alternativas de traducción & Accesible (customización) \\
\hline Modificación de la cantidad de contexto visualizado & Accesible (customización) \\
\hline $\begin{array}{l}\text { Ocultación y modificación de la posición de las ventanas } \\
\text { mostradas }\end{array}$ & Manejable \\
\hline Configuración de colores o tamaño de letra & Manejable y Accesible \\
\hline $\begin{array}{l}\text { Desactivación de las características funcionales que no se } \\
\text { puedan utilizar en esa etapa del proyecto }\end{array}$ & Manejable \\
\hline $\begin{array}{l}\text { Destacado de los términos que estén incluidos en la base } \\
\text { terminológica }\end{array}$ & Usable \\
\hline Denominación relevante de los botones & Manejable y Usable \\
\hline Adicción de cuadros aclarativos desplegables & Manejable y Usable \\
\hline Opción de hacer y deshacer cambios & Usable \\
\hline $\begin{array}{l}\text { Presentación de cuadros de errores con soluciones útiles } \\
\text { y claras }\end{array}$ & Usable \\
\hline $\begin{array}{l}\text { Personalización de la herramienta según gustos, } \\
\text { preferencias o capacidades físicas }\end{array}$ & Accesible (customización) \\
\hline Recopilación datos acerca del proceso de trabajo & Útil y Creíble \\
\hline
\end{tabular}

Tabla 1. Relación entre las características funcionales y los aspectos de interacción

Coral DIEZ CARBAJO Por una mejora de la usabilidad y utilidad en las herramientas de postedición 


\section{CONCLUSIONES}

El paso de mejorar técnicamente las herramientas a aumentar la comodidad humana y la interacción persona-ordenador ha abierto un nuevo camino hacia el diseño de plataformas de postedición centradas en satisfacer a los humanos mientras trabajan. Por ello, motivados por la insatisfacción que expresan los poseditores que han trabajado desde las herramientas híbridas existentes y con el objetivo de desarrollar plataformas de postedición prácticas y fáciles de usar, se han recopilado ciertas características funcionales que resultan de utilidad a partir de las experiencias y opiniones de expertos, investigadores y poseditores profesionales y aplicado los aspectos generales del desarrollo de herramientas usables al caso concreto de las herramientas de postedición asistida.

Este estudio revela que todavía queda mucho por trabajar en la mejora de las herramientas de postedición si se piensa en las necesidades del humano que va a trabajar con ellas, tanto en lo referido a las características funcionales que se podrían incluir antes, durante y después del proceso de postedición y que repercutirían positivamente a la utilidad a las mismas, como con respecto a los aspectos de interacción persona-ordenador que aportarían usabilidad a las herramientas de postedición, lo que aumentaría el rendimiento y la sensación de satisfacción de los poseditores. Por ello, de la gran cantidad de características funcionales útiles y aspectos de interacción que pueden implementarse, se concluye que resulta de vital importancia que se permita customizar dichas herramientas para hacerlas accesibles a cualquier usuario en cualquier momento del proceso de postedición. La personalización de estas herramientas avanzadas de postedición asistida debería abarcar desde los aspectos más básicos, como poder cambiar el color de la interfaz o el tamaño de letra de acuerdo con las limitaciones visuales de cada usuario, hasta la posibilidad de activar o desactivar ciertas características funcionales dependiendo de la etapa del proceso de postedición (creación del proyecto, preedición, entrenamiento de motores, postedición, recopilación de datos del flujo de trabajo, etc.) en el que se encuentre el poseditor o de sus gustos personales, ya que ciertos usuarios pueden considerar que una característica funcional les resulta útil mientras que a otros usuarios le resulte molesta.

Hoy en día, parece que la mayoría de los traductores van a cruzar su camino con la traducción automática con postedición en algún momento de sus carreras: su empleo cada vez es más común para traducir textos con poco valor estético, repetitivos y cargados de terminología especializada, y gracias a ella hacemos frente a encargos más extensos en menos tiempo. De este modo, los resultados del presente estudio sientan las bases de nuevas líneas de investigación; se puede desarrollar una herramienta de postedición que recoja las características aquí detalladas (que esté especialmente diseñada con las necesidades del usuario en mente, que sea fácil de usar y cuyo entorno de trabajo le resulte agradable al poseditor) y estudiar sus repercusiones en el mundo

Coral DIEZ CARBAJO Por una mejora de la usabilidad y utilidad en las herramientas de postedición 
de la traducción automática: rendimiento, productividad, mejora de los resultados en bruto, satisfacción de los poseditores, entre otros. Por ello, puede que sea en el momento de dar una segunda oportunidad a la traducción automática con postedición, apostar por la creación de herramientas que nos sean de utilidad y olvidar las malas experiencias del pasado.

\section{BIBLIOGRAFÍA}

ALLEN, Jeffrey. 2001. «Post-editing: An Integrated Part of Translation Software Program». Language International Magazine 13 (2): 26-29.

BERNTH, Arendse y Claudia GDANIEC. 2001. «MTranslatability». Machine Translation 16, (3): 175218.

DE JeSUS MARTINS, Débora Beatriz y Helena DE MEDEIROS CASELI. 2015. «Automatic machine translation error identification». Machine Translation 29 (1): 1-24.

DILLINGER, Mike. 2014. «Introduction». En Post-Editing of Machine Translation: Processes and Applications, ed. por Sharon O'Brien, Laura Winther Balling, Michael Carl, Michael Simard y Lucia Specia. Newcastle upon Tyne: Cambridge Scholars Publishing, ix-xv.

FLÓREZ, Silvia. 2012. "Catálogo de herramientas para la postedición». Tradumàtica: tecnologies de la traducció. Postedició, canvi de paradigma? 10: 219-229.

GARRETT, Jesse James. 2011. The Elements of User Experience: User-Centered Design for the Web and Beyond, Second Edition. Berkeley, CA: New Riders.

GUERBEROF ARENAS, Ana, Heidi DEPRAETERE y Sharon O'BrieN. 2012. "What we know and what we would like to know about post-editing». Tradumàtica: tecnologies de la traducció. Postedició, canvi de paradigma? 10: 211-218.

GUERBEROF Arenas, Ana. 2008. «Productivity and Quality in the Post-Editing of Outputs from Translation Memories and Machine Translation». The International Journal of Localisation 7 (1): 11-21.

GUTIÉRREZ Y RESTREPO, Emmanuelle y Loïc MARTíNEZ NORMAND. 2010. «Localization and web accessibility». Tradumàtica: tecnologies de la traducció. Localització $i$ web 8. Fecha de acceso 6 de octubre de 2018. http://revistes.uab.cat/tradumatica/article/view/n8-gutierrezmartinez/pdf_21

KAY, Martin. 1997. «It's Still the Proper Place». Machine Translation 12: 35-38.

KUNIAVSKY, Mike. 2012. Observing the User Experience: A Practitioner's Guide to User Research. Waltham, MA: Elsevier.

MELBY, Alan. 1997. «Some Notes on The Proper Place of Men and Machines in Language Translation». Machine Translation 12: 29-34.

MEYER, Kate. 2017. «Comparison Tables for Products, Services, and Features». Nielsen Norman Group. Fecha de acceso 6 de octubre de 2018. https://www.nngroup.com/articles/ comparison-tables/

MOORKENS, Joss y Sharon O'BRIEN. 2017. «Assessing User Interface Needs of Post-Editors of Machine Translation». En Human Issues in Translation Technology, ed. por Dorothy Kenny. Oxford: Routledge, 109-130.

Coral DIEZ CARBAJO Por una mejora de la usabilidad y utilidad en las herramientas de postedición
CLINA

vol. 4-2, December 2018, 123-140

elSSN: 2444-1961

Ediciones Universidad de Salamanca - CC BY-NC-ND 
MOORKENS, Joss, Sharon O'BRIEN y Joris VREEKE. 2016. «Developing and testing Kanjingo: A mobile app for post-editing». Tradumàtica: tecnologies de la traducció. Traducció $i$ dispositius mòbils 14: 58-66.

MORVILLE, Peter. 2004. «User Experience Design». Semantic Studios. Fecha de acceso 6 de octubre de 2018. http://semanticstudios.com/user_experience_design/

NiELSEN, Jakob. 1995. "10 Usability Heuristics for User Interface Design». Nielsen Norman Group. Fecha de acceso 6 de octubre de 2018. https://www.nngroup.com/articles/tenusability-heuristics/

NiELSEN, Jakob. 2012. «Usability 101: Introduction to Usability». Nielsen Norman Group. Fecha de acceso 6 de octubre de 2018. https://www.nngroup.com/articles/usability-101introduction-to-usability/

NUNES VIEIRA, Lucas y Lucia SPECIA. 2011. "A Review of Translation Tools from a Post-Editing Perspective». Third Joint EM+/CNGL Workshop Bringing MT to the User: Research Meets Translators (JEC 2011). Fecha de acceso 6 de octubre de 2018. http://www.mt-archive. info/JEC-2011-Vieira.pdf

O'BRIEN, Sharon, Laura WINTHER BALLING, Michael CARL, Michael SIMARD y Lucia SPECIA. 2014. «Foreword». En Post-Editing of Machine Translation: Processes and Applications, ed. por Sharon O'Brien, Laura Winther Balling, Michael Carl, Michael Simard y Lucia Specia. Newcastle upon Tyne: Cambridge Schoolars Publishing, vii-viii.

O'BRIEN, Sharon. 2006. «Pauses as Indicators of Cognitive Effort in Post-Editing Machine Translation Output». Across Languages and Cultures 7, (1): 1-21.

O'BRIEN, Sharon. 2012. «Towards Predicting Post-Editing Productivity». Machine Translation 25: $197-215$.

O'BRIEN, Sharon y Joss MOORKENS. 2014. «Towards intelligent post-editing interfaces». FIT XXth World Congress 2014, Berlin, Germany. Fecha de acceso 6 de octubre de 2018. http:// doras.dcu.ie/20136/

RAMíREZ POLO, Laura. 2012. «Los lenguajes controlados y la documentación técnica: mejorando la traducibilidad». Tradumàtica: tecnologies de la traducció. Postedició, canvi de paradigma? 10: 192-204.

REINKE, Uwe. 2013. "State of the Art in Translation Memory Technology". Translation: Computation, Corpora, Cognition 3 (1): 27-48.

RICO PÉREZ, Celia. (s.f.). «Introducción a la Traducción Controlada». A blog on Translation and Technology (T2) (blog). Fecha de acceso 6 de octubre de 2018. www.celiarico.wordpress.com

ROBERT, Anne-Marie. 2010. "La post-édition : l'avenir incontournable du traducteur ?». Traduire 222: 137-144.

RODRíGUEZ VÁZQUEZ, Silvia y Jesús TORRES DEL REY. 2014. «Fostering accessibility through web localization». Multilingual Magazine 147, 25 (7): 32-37.

SÁNCHEZ-MARTíNEZ, Felipe. 2012. «Motivos del creciente uso de traducción automática seguida de postedición». Tradumàtica: tecnologies de la traducció. Postedició, canvi de paradigma? 10: $150-156$.

SILVA, Roberto. 2014. «Integrating Post-Editing MT in a Professional Translation Workflow». En Post-Editing of Machine Translation: Processes and Applications, ed. por Sharon O'Brien, Laura Winther Balling, Michael Carl, Michael Simard y Lucia Specia. Newcastle upon Tyne: Cambridge Schoolars Publishing, 24-50.

Coral DIEZ CARBAJO

Por una mejora de la usabilidad y utilidad en las herramientas de postedición 
SUKHOLEYSTER, Oleksandr. 2014. "Task-Driven User Interfaces», UX matters. Fecha de acceso 6 de octubre de 2018. https://www.uxmatters.com/mt/archives/2014/12/task-driven-userinterfaces.php

TAUS. 2010. «MT Post-editing Guidelines». Fecha de acceso 6 de octubre de 2018. https:// es.taus.net/academy/best-practices/postedit-best-practices/machine-translation-postediting-guidelines

TORRES DEL REY, Jesús y Lucía MORADO VÁZQUEZ. 2015. «XLIFF and the Translator:Why Does it Matter?». Tradumàtica: tecnologies de la traducció. Postedició, canvi de paradigma? 13: 584-607.

WINOGRAD, Terry. 1997. «From Computing Machinery to Interaction Design». En Beyond Calculation: The Next Fifty Years of Computing, ed. por Peter Denning y Robert Metcalfe. Berlín: Springer-Verlag, 149-162. 